\title{
Intervención en la discapacidad intelectual y trastorno mental asociado en el adulto: de la intervención sistémica, el equipo interdisciplinar y la coherencia de las intervenciones.
}

Intervention in intellectual disabilities and mental disorder in adults associated: systemic intervention, the interdisciplinary team and consistency of assistance.

\author{
Mª Jesús Montes Lozano a, Pilar Paños Núñez ${ }^{\text {. }}$ \\ ${ }^{a}$ Servicio de Salud Mental. Complejo Hospitalario Universitario, Albacete, España. ${ }^{b}$ Psicóloga de la Aso- \\ ciación para la Atención del Retraso Mental-ASPRONA, Albacete, España.
}

Correspondencia: $M^{a}$ Jesús Montes Lozano (mjmontes@sescam.jccm.es)

Recibido: 01/02/2014; aceptado: 30/06/2014

\begin{abstract}
RESUMEN: Toda reflexión en la línea de mejorar el abordaje en la Discapacidad Intelectual (DI) desde la visión ecológica y biopsicosocial, debe incluir el estudio de las relaciones significativas del individuo, tanto las familiares como las del contexto, como forma de entender las capacidades que aún estando conservadas, la disfunción relacional las aminora.

En el abordaje sistémico se lee la patología desde la relación de los sistemas con los que la persona está en contacto, formulando hipótesis que pueden confirmarse o refutarse durante el proceso terapéutico. El trabajo compartido con el grupo familiar permite organizar los recuerdos como un puzzle que de sentido a la propia historia.

Se describe el programa de intervención en Discapacidad intelectual y trastornos de conducta en Asprona de Albacete, las estrategias de intervención basadas en trabajo interdisciplinar, análisis funcional de la conducta, evaluación familiar e importancia del profesional de referencia como vínculo privilegiado.

Se destaca la importancia de la figura nutricia o vínculo familiar privilegiado, patrones de relación redundantes como triangulaciones manipuladoras, sobreindulgencia y parentificación sobre los que intervenir con la familia y en el contexto.
\end{abstract}

PALABRAS CLAVE: Discapacidad intelectual, Terapia familiar, Terapia combinada.
ABSTRACT: Any reflection on the line to improve the approach to the Intelectual Disability (ID) from the ecological and biopsychosocial view, should include consideration of the significant relationships of the individual, the family as much context as a way to understand the capabilities while still conserved, the relational dysfunction makes it smaller.

In the systemic approach pathology is read from the ratio of the systems with which the person is contacted by formulating hypotheses that can be confirmed or refuted during the therapeutic process. The share in the household work to organize the memories like a puzzle that sense of history itself. Intervention program in intellectual disability and behavioral disorders in Asprona $\mathrm{AB}$, intervention strategies based on interdisciplinary work, functional behavior analysis, family assessment and importance of professional reference and special link is described.

The importance of nurturing figure or privileged family ties, redundant patterns of relationship as manipulative triangulations, overindulgence and parentification on to intervene with the family and the context is emphasized.

KEY WORDS: Intellectual Disability, Family Therapy, Combined Modality Therapy.

Agradecimiento:

A la dirección y profesionales de La Asociación para la Atención a Personas con Discapacidad Intelectual y sus familias (ASPRONA)-Albacete, por su ayuda en la recogida de datos, confianza y autonomía en la elección y diseño de estrategias de intervención. 


\section{Introducción}

Dentro de un marco de reflexión, se trata de un estudio descriptivo cuyo objetivo es en primer lugar trasladar la experiencia de atención a los usuarios en régimen de internado con discapacidad intelectual (DI) y trastorno mental, seguidos desde el año 1995 en la Asociación para la atención a la personas con discapacidad intelectual y sus familias (ASPRONA) de Albacete tanto en la vertiente del diagnóstico como de la intervención desde varias disciplinas y a través de un equipo interdisciplinar compuesto por psiquiatra, psicólogo, enfermería y Trabajador social y de forma puntual con otros miembros de la red asistencial.

Se encuentra en elaboración estudio estadístico que permita objetivar resultados desde la intervención descrita.

El equipo multidisciplinar es eje central. Este equipo permite ensanchar el marco teórico de referencia, el análisis del caso, un mayor ajuste a la hora de elegir la estrategia de intervención y como objetivo a perseguir el propiciar el trabajo activo por parte de los intervinientes directos con el paciente (cuidadores, enfermería, psicología, monitores) y su familia.

Desde la Terapia Sistémica se estudia el síntoma "problemas de conducta" y se intenta construir un mapa relacional que ayude a descifrar los patrones disfuncionales que perpetúan la presencia del síntoma.

Una de las críticas de las clasificaciones al uso, DSM-IV-TR (1) y CIE-10 (2), ha sido la denominación "Retraso mental", centrada en el defecto, siendo modificada en el DSM-5 como Discapacidad Intelectual , con revisión de criterios diagnósticos más adaptados a este grupo de pacientes (3).

Como indica Verdugo (4-5) la DI se debe plantear con un modelo ecológico y contextual, en el cual se debe integrar la persona y el contexto.

Toda reflexión en la línea de mejorar el abordaje en la DI desde la visión biopsicosocial, sería conveniente incluir el estudio de las relaciones significativas del individuo, tanto las familiares como las del contexto, como forma de entender las capacidades que aún estando conservadas, la disfunción relacional las aminora. A su vez en una mayoría de casos confunde a los profesionales, patologizando con etiqueta de "trastorno mental" la expresión comportamental del malestar. De ahí que el diagnóstico sistémico pueda utilizarse como una herramienta en la línea difusa entre patología mental y relación disfuncional.

El modelo médico puede ser de utilidad para identificar y tratar las enfermedades, pero muchos problemas de conducta no serán consecuencia exclusiva de la patología.

Desde el modelo médico-psiquiátrico no es incompatible complementar la evaluación con el estudio psicológico, desde la modalidad o escuela en el esté formado el profesional. En el abordaje sistémico se lee la patología desde la relación de los 
ORIGINALES Y REVISIONES

sistemas con los que la persona está en contacto, formulando hipótesis que pueden confirmarse o refutarse durante el proceso terapéutico (6).

El nacimiento de un hijo discapacitado pondrá a prueba la estructura familiar. Su larga duración pone en interacción al paciente, su familia y el sistema de cuidados.

Ningún estilo familiar individual es inherentemente normal o anormal. Una adecuada jerarquía generacional y líneas claras de autoridad parental son esenciales para un funcionamiento óptimo. La distribución igualitaria del poder en la unidad conyugal-parental es crucial para el equilibrio parento-filial. Alcanzar fortaleza familiar requiere fronteras y subsistemas claros, si bien flexibles, a fin de movilizar pautas de respuesta alternativas cuando los desafíos de la enfermedad y discapacidad someten a la familia a una situación de estrés.

La terapia familiar se apoya en el concepto de regulación y organización circular. El comportamiento de cada individuo se comprende en el contexto en el que aparece y el síntoma surge para suministrar equilibrio (7).

No se considera que los miembros de una familia poseen ciertas características innatas, sino que manifiestan una conducta en relación con la conducta de otros. Se considera que ninguna persona tiene el control unilateral sobre ninguna otra, el control está en el modo en que el circuito se organiza y continúa operando (8).

Linares JL (9) hace referencia al estilo terapéutico propio, siendo los factores que lo integran: espontaneidad, estilo personal, responsabilidad, compromiso, ética, creatividad, flexibilidad, experiencia y humildad son ingredientes para un buen terapeuta.

Boscolo, L (10) apoya el concepto de integración de técnicas, no como la utilización indiscriminada de diversos modelos teóricos, sino a través de un modelo teórico bien experimentado.

$\mathrm{El}$ interés fundamental del terapeuta en la DI con problemas de conducta, es el examen detenido del funcionamiento de la conducta y el modo en que la función de un comportamiento aislado se conecta con otro y modifica las pautas de relación.

El espacio pragmático va a ser definidor de la naturaleza de las relaciones interpersonales en el paciente con DI, puesto que a través de él aparecerá la expresión sintomatológica, por ello los problemas conductuales se deben de entender como el síntoma a explorar inmerso en la dinámica familiar, explorando la funcionalidad que ejerce en el sistema familiar.

A pesar de las deficiencias cognitivas del paciente, el abordaje de las emociones que surgen en el paciente y la familia, facilitan el cambio y dan la posibilidad de anular el comportamiento anómalo como comunicación.

Una intervención orientada al cambio debe procurar "una experiencia emocional correctiva", abriendo la vía a un cambio emocional, cognitivo y comportamental (11). 
Material y Métodos

Evaluacion del programa en discapacidad intelectual y problemas de conducta. Asprona- $a b$

ASPRONA de Albacete, ante la alta frecuentación de urgencias psiquiátricas por los problemas conductuales que se generaban en los diferentes dispositivos, la falta de disponibilidad y retraso en las revisiones en Salud Mental pública, inició un Programa de Atención Especializada en Salud Mental, que lleva más de 15 años funcionando, en el que el psiquiatra forma parte del equipo de intervención, cuyo objetivo final es mejorar aspectos clínico-conductuales que propicien una conducta adaptada que integre al usuario en el contexto.

Se expondrá en la presentación los usuarios atendidos en el programa y patología psiquiátrica comórbida.

Los usuarios son atendidos según sus necesidades en:

1. Centro ocupacional (externado)

2. Pisos supervisados

3. Residencia comunitaria

4. Miniresidencia para gravemente afectados

En las tablas se aprecia el elevado porcentaje de pacientes en tratamiento psiquiátrico, especialmente, los pacientes internados, así como la mayor prevalencia de T. Control de impulsos y del espectro psicótico.

Tabla 1

Pacientes en tratamiento psiquiátrico en Centro ocupacional

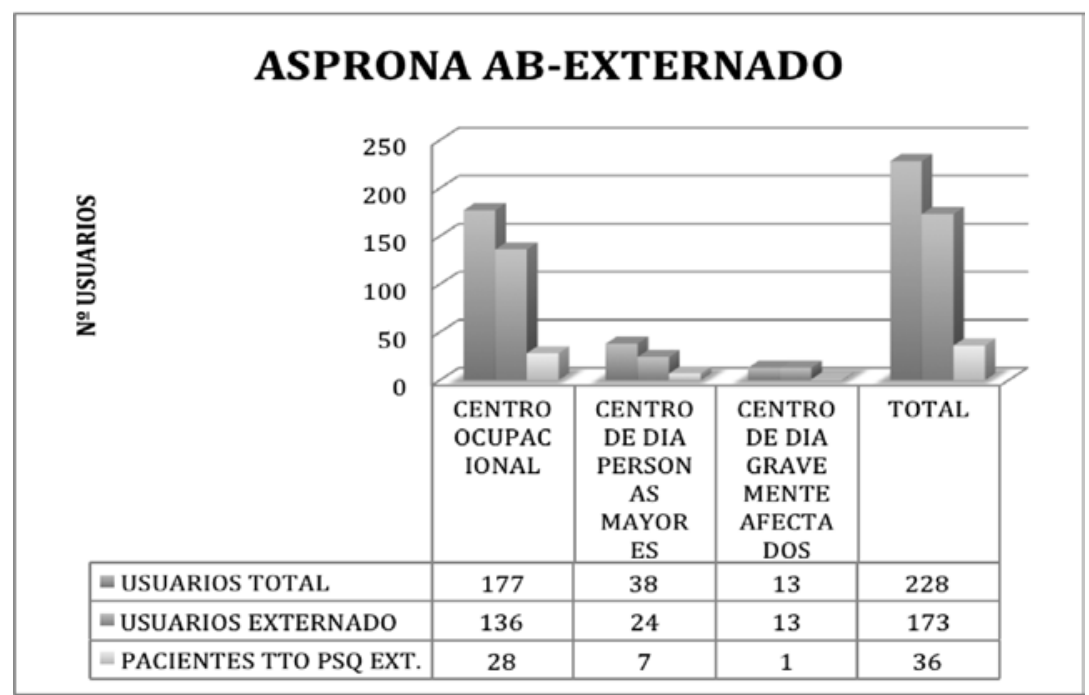


ORIGINALES Y REVISIONES

Tabla 2

Pacientes en tratamiento psiquiátrico en Viviendas e Internado

ASPRONA AB INTERNADOVIVIENDAS

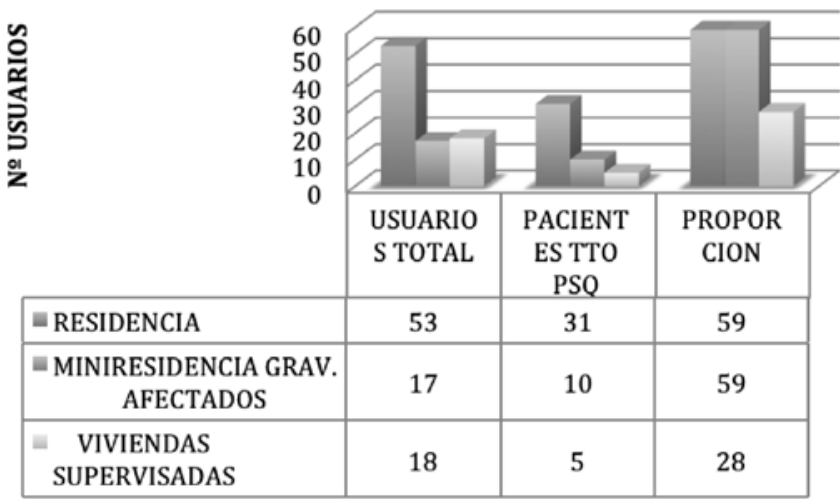

Tabla 3

Diagnóstico psiquiátrico usuarios residencia comunitaria

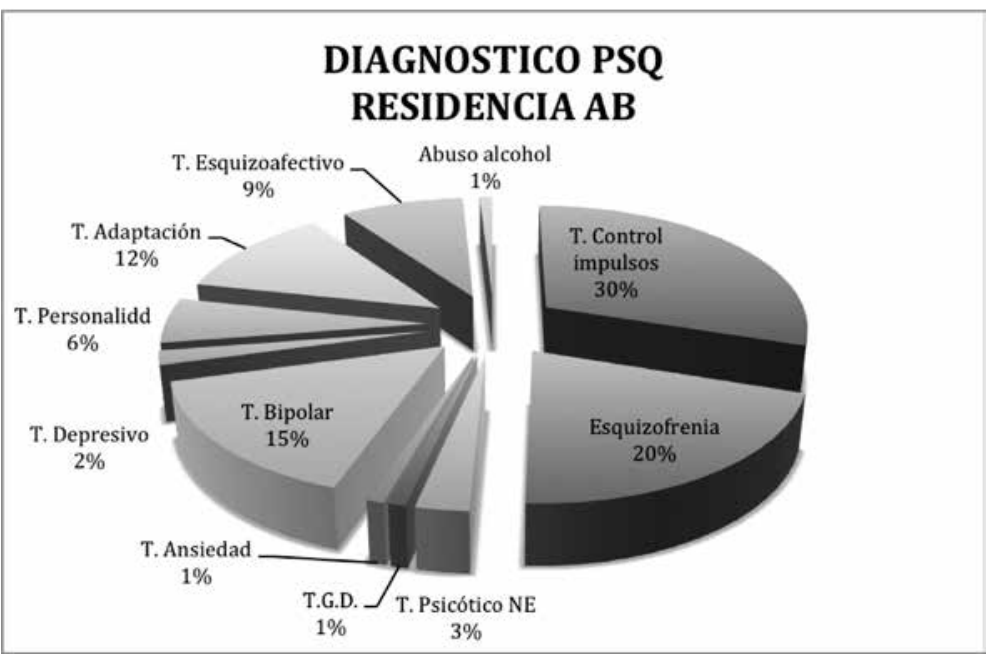


Diagnóstico psiquiátrico usuarios miniresidencia gravemente afectados

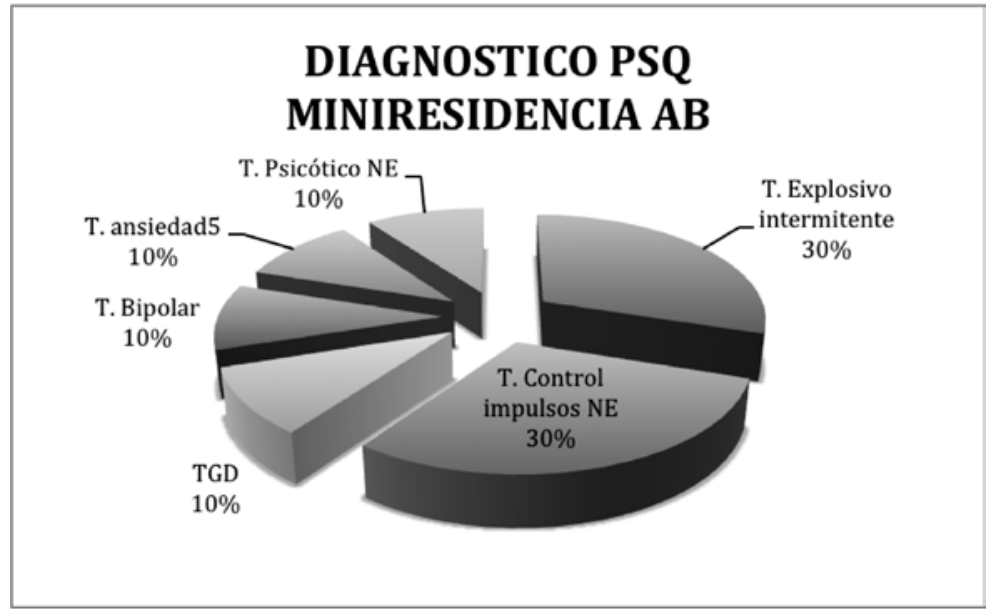

Destacaría resumidamente las características de este PROGRAMA:

a. Trabajo multidisciplinar:

i. Valoración e intervención conjunta directa con el paciente psiquiatrapsicóloga en casos complejos.

ii. Intervención directa con usuarios por parte de profesionales de Asprona en caso de menor gravedad.

iii. Abordaje complementando modelos de abordaje: biológico, social, evaluación funcional de la conducta, hipotetización relacional desde la Terapia Sistémica

iv. Inclusión de DUE y TS en sus ámbitos de competencia

v. Importancia de la figura del referente del paciente, como forma de vínculo preferente.

b. Psiquiatra como consultor, no como integrante permanente del equipo.

c. Accesibilidad al psiquiatra ante situación de crisis.

d. Trabajo con Plan Terapéutico individualizado. Se trabaja individualmente con llamadas teléfono, visitas, salidas puntuales, salidas al domicilio sin o con pernoctación, se elige momento, personas, etc.

e. Evaluación más allá de la inclusión en un grupo diagnóstico, sino el estudio de su mapa relacional que incluye las relaciones con profesionales, familia y demás usuarios

f. Mantenimiento del equipo y seguimiento continuado.

Diversos modelos en nuestro país apuntan sobre las ventajas de crear una unidad especializada, incluida en la Sanidad Pública, con personal cualificado y programas específicos (12). 
ORIGINALES Y REVISIONES

\section{Discusión}

Desde el modelo sistémico, se indican las técnicas y estrategias utilizadas en este programa:

1. Se mantiene la formulación de hipótesis que permite conectar informaciones, significados y acciones en un estudio transgeneracional y diacrónico. Es similar a un fotograma familiar en el que se detiene en momentos cruciales del ciclo vital familiar.

2. Se complementa la visión circular y lineal, teniendo en cuenta los puntos de vista de los miembros intervienen en la terapia.

3. Se amplia el foco no solo a la relación, sino también al individuo.

4. Importancia del trabajo emocional con los pacientes y las familias, la neutralidad no debe impedir abordar los sentimientos generados en las sesiones.

5. Se adapta el estilo terapéutico y combinan técnicas de las diferentes escuelas sistémicas, para adaptarlas a las características de la familia, pudiéndose integrar el modelo de la Escuela de Milán, el estructural (Minuchin) y el estratégico (Nardone).

6. Se interviene tanto a nivel familiar, con subsistemas, con el individuo, o bien con intervinientes de apoyo del paciente.

7. La elección de miembros con los que se interviene es flexible y buscando la estrategia de que acudan los convocados.

8. En todos los casos se estudia la demanda previamente, se establecen hipótesis de partida y se inicia el abordaje partiendo de la información aportada por referentes y conocedores del problema.

9. Con el repertorio básico de técnicas, en este caso bajo el prisma sistémico, se podrá trabajar indistintamente en sesiones individuales, familiares, subsistémicas o de red. El resultado final dependerá más de la coherencia del enfoque en su conjunto que de la naturaleza de cada opción correcta.

El punto de partida es que los casos con mayores necesidades, atienden a un perfil en el que la conducta disruptiva es su modo de relación, es la estrategia elegida para tener un lugar en la relación con los demás, suelen ser mantenidas en el tiempo, inflexibles y resistentes al cambio. A través del síntoma que presenta se obtiene o se pretenden beneficios secundarios, no se encuentran otras vías de comunicación, es la fase en la que el síntoma ha desarrollado "poder relacional" en el contexto en el que aparece.

La familias han pasado por múltiples dificultades, en muchas ocasiones impotentes y vulnerables, responden con patrones inflexibles, visto como resistencia al cambio por los profesionales y a su vez entendido como funcionamiento homeostático que preserve su identidad familiar. 
Según Bergman (13) cuanto más grave y crónico es el síntoma, más resistente al cambio será la familia.

El trabajo con las familias, parte de sus competencias y puntos fuertes, afrontando a través del desarrollo de la terapia los momentos vitales de vulnerabilidad y el recorrido de momentos de crisis que a través del síntoma o del trastorno de conducta aportaron un pseudoestabilidad al sistema.

$\mathrm{Al}$ paciente se le posiciona como persona competente con potencial de cambio, en un discurso asertivo, estableciendo una relación de confianza que le haga permeable.

Se trabaja con la hipotetización del caso, dividiendo la fase de la intervención en tres partes

1. Acomodación. Búsqueda de empatizar, creando un clima emocional aduado, flexible, acomodada al paciente y/o familia.

2. Evaluación

3. Desarrollo de la terapia. Se interviene desde el marco teórico de la terapia sistémica, pero acomodado a la tipología de la familia, de ahí que también se introducen técnicas estratégicas, especialmente útiles en las Terapias Breves y en DI a través de rituales y prescripciones (Nardone, 2011).

4. Finalización.

En aquellos casos que se trabaja con el paciente, los objetivos comunes, se establecerían (14)

- Importantes para el paciente

- Formulados de manera positiva

- Formulados a partir de la visión del paciente

- Objetivos existenciales concretizados en pequeños

- hechos realizables

- Flexibles y adaptables

- Formulados de manera interaccional

- Presentados como necesitando un esfuerzo

Ante la presencia de rechazo o resistencia a la intervención, se pueden incluir diversas estrategias:

i. No adoptar actitud defensiva

ii. Facilitar argumentos del o los miembros

iii. Dejar abiertas posibilidades futuras

iv. Aportar información que solicite

v. Si solicitan abordaje individual, recoger información con la familia

vi. Clarificar las metas u objetivos 
vii. Articular respuestas flexibles

viii. Evitar culpabilizar a la familia, trasladando las limitaciones de los profesionales que atienden al usuario y ofrecer con humildad la impotencia ante los avances.

\section{Conclusiones}

Para finalizar resaltar aspectos que atienden a redundancias observadas a lo largo del trabajo con los pacientes y familias con DI:

$\sqrt{ }$ En el marco de necesidad de cuidados y limitación a la autonomía, se establece una relación de dependencia emocional respecto a las figuras amorosas primarias o nutricias

$\sqrt{ }$ Estas figuras pueden pertenecer a la familia nuclear ( con frecuencia madre, hermanos) o la familia extensa (abuelos,...) nos darán la llave que permita al terapeuta intervenir hacia el cambio y normalización relacional

$\sqrt{ }$ En la hipotetización sobre la funcionalidad del síntoma hay que explorar juego relacional en curso, del que forma parte primordial la figura primaria nutricia, explorando:

- Posibles triangulaciones manipuladoras, que pueden ocasionar juegos perversos contra los cuidadores sustitutivos (profesionales de las instituciones)

- Tipo de relación, si por el paciente es vivida como rechazo o desconfirmación, el síntoma es utilizado como vendeta ante la vivencia de abandono.

$\sqrt{ }$ Cuando más grave y crónico es el síntoma, más difícil modificarlo, va difuminando el objetivo por el que se inició, tomando autonomía propia a través del poder relacional que ejerce, siendo utilizada por el paciente de forma ampliada a todo el abanico relacional

$\sqrt{ }$ Tanto la parentificación del individuo en momentos vulnerables de la familia, como pautas redundantes de sobreindulgencia hacia él, son disfunciones en la estructura del subsistema parento-filial, que pueden favorecer la aparición del síntoma, si la persona siente que ha perdido la relación privilegiada.

$\checkmark$ Las dificultades de encontrar pautas relacionales nuevas del paciente, el ser conocedor de las ventajas que le otorga el síntoma, el temor a perder estos beneficios, la falta de autonomía y el deber de la familia de proveer de cuidados son algunos de los factores que marcarán la resistencia al cambio. 
ORIGINALES Y REVISIONES

\section{BIBLIOGRAFÍA}

(1) American Psychiatric Association. DSM-IV-TR. Manual de Diagnóstico Estadístico de los Trastornos Mentales. Barcelona: Masson, 2002.

(2) Organización Mundial de la Salud. Clasificación Internacional Estadística de Enfermedades y problemas relacionados de la salud (CIE-10). Ginebra: OMS, 2001.

(3) American Psychiatric Association. DSM-5. Guía de consulta de los criterios diagnósticos del DSM-5. Madrid: Panamericana, 2013.

(4) Verdugo, MA. Análisis de la definición de discapacidad intelectual de la AAMR de 2002. Siglo Cero 2003, 34 (1): 5-20.

(5) Verdugo M.A., Schalock R. Últimos avances en el enfoque y concepción de las personas con discapacidad intelectual. Siglo Cero 2010, 41(4): 7-21.

(6) F. Rojero C, Súarez T; Vázquez, P. La entrevista sistémica: De la conducción a la conclusión. Rev. Psicoterapia y Psicosomática 1983, nº 7.

(7) 14-Watzlawich P, Beavin J, Jackson, D. Teoría de la Comunicación humana. Barcelona: Herder; 1989.

(8) Pap P. El proceso de cambio. Barcelona: Paidós, 1991.

(9) Linares , JL; Ceberio, M. Ser y hacer en Terapia Sistémica. Barcelona: Paidós, 2005.

(10) Boscolo L, Bertrando P. Terapia Sistémica individual. Buenos Aires: Amorrortu, 2008.

(11) Nardone G, De Santis, G. Pienso, luego sufro. Barcelona: Paidós, 2012

(12) García Ibañez, J; Feliu, T, Usón M, y otros. Trastornos invisibles. Las personas con discapacidad intelectual y trastornos mentales de conducta. Siglo Cero, 40,(2), 2009, 38-60.

(13) Bergman, J. Pescando Barracudas. Barcelona: Paidós, 1991.

(14) Isebaert, L. La terapia sistémica centrada en soluciones. Seminario realizado por el Centro de estudios Sistémicos de AESFAHU. Madrid, marzo, 2011. 